\section{Der Grundsatz der persönlichen Leistungserbringung in der vertragsärztlichen Versorgung, insbesondere bei kooperativer Zusammenarbeit (Teil 2)}

\section{Einleitung}

In dem ersten Teil dieses Beitrags wurde die Ausprägung des Grundsatzes der persönlichen Leistungserbringung in der vertragsärztlichen Versorgung dargestellt. ${ }^{1}$ Dabei wurden die Möglichkeiten der Delegation von ärztlichen sowie nichtärztlichen Leistungen umrissen und die Besonderheiten in den Strukturen von Berufsausübungsgemeinschaften (BAG), Medizinischen Versorgungszentren (MVZ) und Filialen in den Blick genommen.

Der hier folgende zweite Teil wird sich mit den Möglichkeiten einer zulässigen Vertretung und der Durchbrechung des Grundsatzes der persönlichen Leistungserbringung bei kooperativer Zusammenarbeit in BAG, MVZ und Filialen sowie Fragen der Abrechnung von vertragsärztlichen Leistungen, die unter Mitwirkung mehrerer Ärzte erbracht wurden, befassen.

\section{Vertretung in BAG, MVZ und Filialen}

Gemäß §32 Abs. 1 S. 1 Ärzte-ZV hat der Vertragsarzt die vertragsärztliche Tätigkeit persönlich in freier Praxis auszuüben. Bei Krankheit, Urlaub oder Teilnahme an ärztlicher Fortbildung oder an einer Wehrübung kann er sich innerhalb von zwölf Monaten bis zur Dauer von drei Monaten vertreten lassen. Die Vertretungsgründe sind für die vertragsärztliche Versorgung abschließend geregelt. Dies gilt gemäß §32b Abs. 6 S. 1 , 2. HS Ärzte-ZV für angestellte Ärzte gleichermaßen.

Die Kassenärztlichen Vereinigungen unterscheiden in ihrer jeweiligen Verwaltungspraxis zwischen „echter“ Vertretung nach $\S 32$ Abs. 1 Ärzte-ZV, sogenannter „unechter" Vertretung innerhalb einer Praxis, einer BAG oder eines MVZ sowie der kollegialen Vertretung in der Praxis des Vertreters.

1 Vgl. Fortschr Röntgenstr 2019; 191: 580 - 584.
Ein „echter“ Vertreter im Sinne des Vertragsarztrechts ist ein Arzt, der nicht in der Praxis, der BAG oder dem MVZ des Vertretenen tätig ist und entweder als Arzt (auch als angestellter) in einer anderen Praxis, einer anderen BAG oder einem anderen MVZ an der vertragsärztlichen Versorgung teilnimmt oder nicht (auch nicht als angestellter Arzt) an der vertragsärztlichen Versorgung teilnimmt.

Im Umkehrschluss ist ein „unechter“ Vertreter ein interner Vertreter, der entweder als Vertragsarzt gemeinsam mit dem Vertretenen im Rahmen einer gemeinsamen Berufsausübung an der vertragsärztlichen Versorgung teilnimmt oder als angestellter Arzt in der Praxis, der BAG oder des MVZ des Vertretenen gemäß §32b Abs. 1 ÄrzteZV, §95 Abs. 9 SGB V tätig ist.

Die verschiedenen Kassenärztlichen Vereinigungen beantworten die Frage, ob eine „echte“ oder eine „unechte“ Vertretung vorliegt, in Abhängigkeit der Status von Vertreter und Vertretenem (Vertragsarzt oder angestellter Arzt) jedoch teilweise abweichend, sodass diesbezüglich stets auf die Verwaltungspraxis der jeweils zuständigen Kassenärztlichen Vereinigung abzustellen ist.

Eine kollegiale Vertretung (vgl. §20 Abs. 1 MBO-Ä) liegt auch nach einhelliger Auffassung der Kassenärztlichen Vereinigungen vor, wenn ein Vertragsarzt in seiner eigenen Praxis einen anderen Vertragsarzt vertritt.

\section{„Echte“ Vertretung}

Ein Vertragsarzt hat eine eigene, bzw. die Abwesenheit eines angestellten Arztes von mehr als einer Woche der für ihn zuständigen Kassenärztlichen Vereinigung nach $\S 32$ Abs. 1 S. 4 Ärzte-ZV anzuzeigen.

Als „Nicht-Vertragsarzt“ kann jeder beliebige Arzt als Vertreter beauftragt werden, der als approbierter Facharzt über die erforderlichen Qualifikationen verfügt (vgl.

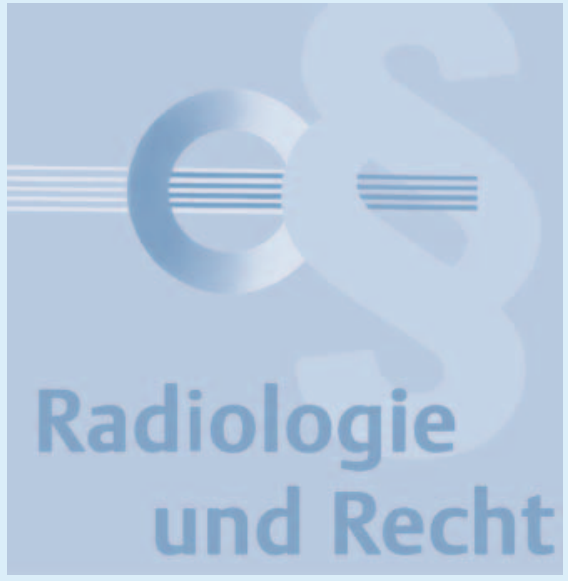

§§ 32 Abs. 1 S. 5,3 Abs. 2 Ärzte-ZV), mithin auch ein angestellter Arzt der Praxis, der BAG oder des MVZ, der lediglich an der privatärztlichen Versorgung teilnimmt.

Der „Nicht-Vertragsarzt“, der als echter Vertreter tätig wird, muss ausreichend qualifiziert sein. Er muss über eine abgeschlossene Facharztausbildung in dem Fachgebiet des Vertretenen verfügen. Qualitätsgebundene Leistungen darf er nur erbringen, wenn er ausreichend qualifiziert ist und über die erforderlichen Abrechnungsgenehmigungen gemäß § 135 Abs. 2 SGB V verfügt bzw. deren Voraussetzungen zumindest theoretisch erfüllt. Nicht vertragsärztlich tätige Ärzte müssen die erforderlichen Qualifikationen über Testate nachweisen können.

Der „echte“ Vertreter kann ein Vertragsarzt aus demselben oder einem anderen KVBezirk sein. Die „echte“ Vertretung eines externen Vertragsarztes liegt dann vor, wenn der externe Vertragsarzt die Vertretung in den Praxisräumen des Vertretenen durchführt.

\section{Abrechnung der „echten“ Vertretung}

Die von dem „echten“ Vertreter erbrachten Leistungen sind als Vertreterleistungen zu kennzeichnen. Es ist das Muster 19 der Anlage 2 BMV-Ä („Notfall-/Vertretungsschein“) zu verwenden und auf die Vertretung hinzuweisen.

Bei einer „echten“ Vertretung in der Praxis des Vertretenen sind die Vertreterleistungen mit der Lebenslangen Arztnummer (LANR) des Vertretenen zu kennzeichnen; unabhängig davon, ob es sich bei dem 
Vertreter um einen Arzt mit dem Recht zur Teilnahme an der vertragsärztlichen Versorgung oder um einen „Nicht-Vertragsarzt" handelt.

Im Rahmen der kollegialen Vertretung sind die Leistungen unter der LANR des (kollegialen) Vertreters über dessen Praxis abzurechnen.

\section{„Unechte“ Vertretung}

Die Vertretung eines Vertragsarztes bzw. angestellten Arztes innerhalb einer Praxis, einer BAG oder eines MVZ durch einen internen Vertreter, d. h. einen Vertragsarzt oder einen angestellten Arzt derselben Praxis, derselben BAG bzw. desselben MVZ, stellt (je nach Verwaltungspraxis der zuständigen Kassenärztlichen Vereinigung) eine sogenannte „unechte“ Vertretung dar, auf die die Vertretungsregelungen in $\S 32$ Abs. 1 Ärzte-ZV keine Anwendung finden.

Diese Rechtsauffassung resultiert letztlich aus einem Urteil des Bundessozialgerichts (BSG), in dem dieses klargestellt hat, dass eine Behandlung durch einen anderen Arzt einer BAG (einer Praxis oder eines MVZ) nicht als Vertretung im Sinne von $\S 32$ Abs. 1 Ärzte-ZV anzusehen ist. ${ }^{2}$

Zu beachten ist, dass von einigen Kassenärztlichen Vereinigungen die Auffassung vertreten wird, dass jegliche interne Vertretung innerhalb einer BAG oder eines MVZ, unabhängig vom Umfang des Versorgungsauftrags des Vertreters, als „unechte Vertretung “ anzusehen ist, obwohl ein Vertragsarzt oder angestellter Arzt, dem kein voller Versorgungsauftrag übertragen wurde, über ausreichende Kapazitäten verfügt (exklusive der vertragsärztlichen Verpflichtungen), um Vertretungen außerhalb und innerhalb der jeweiligen BAG bzw. des MVZ zu leisten. Entsprechend nehmen andere Kassenärztliche Vereinigungen in dem Fall, in dem einem intern vertretenden Arzt kein voller Versorgungsauftrag übertragen ist, einen Fall der „echten“ Vertretung nach $\S 32$ Abs. 1 Ärzte-ZV an, soweit der Vertreter über den Umfang des ihm übertragenen Versorgungsauftrags

2 BSG, Urteil vom 14.12.2011, Az.: B 6 KA 31/ $10 \mathrm{R}=$ SozR $4-2500 \S 106 \mathrm{Nr} 8$. hinaus in Vertretung eines Kollegen vertragsärztliche Leistungen erbringt.

Eine weitere Besonderheit besteht im Zusammenhang mit der Vertretung in den sogenannten „Versorgerzweigpraxen“, von denen im ersten Teil dieses Beitrags bereits die Rede war. Diesbezüglich gehen die Kassenärztlichen Vereinigungen wohl überwiegend davon aus, dass eine „unechte“ Vertretung nur an den Betriebsstätten anzunehmen ist, an denen eine vertragsärztliche Tätigkeit des Vertreters zulässig bzw. genehmigt ist. Im Übrigen wird der vertretende Arzt auch innerhalb „seiner“ BAG oder „seines“ MVZ Leistungen als „echter“ Vertreter erbringen können.

Die Frage, ob ein Fall der „echten“ oder der „unechten“ Vertretung vorliegt, ist auch deshalb relevant, weil der „unechte“ Vertreter kein Vertreter im Sinne der Ärzte-ZV ist und folglich für sämtliche Leistungen, die er erbringt, bzw. an deren Erbringung er beteiligt ist, über sämtliche für die Abrechnung dieser Leistung erforderlichen Abrechnungsgenehmigungen verfügen muss.

\section{Abrechnung der „unechten“ Vertretung}

Soweit es sich bei einer internen Vertretung nach der Verwaltungspraxis der zuständigen Kassenärztlichen Vereinigung nicht um eine Vertretung im Sinne der Ärzte-ZV handelt, sind die Leistungen, die im Rahmen der Vertretung erbracht werden, mit der eigenen LANR des Leistungserbringers zu kennzeichnen.

Die Leistungen als „unechter“ Vertreter sind jedoch nicht als Vertretungsleistung zu kennzeichnen, da keine Vertretung im Sinne des BMV-Ä vorliegt. Der „unechte“ Vertreter muss seine Leistungen mit seiner eigenen LANR kennzeichnen.

\section{Vertretung eines PVA}

Ein Programmverantwortlicher Arzt im Rahmen des Mammographie-ScreeningProgramms (PVA) kann sich gemäß §32 Abs. 1 Ärzte-ZV vertreten lassen. Es gilt gemäß §32 Abs. 4 der Anlage 9.2 BMV-Ä die Besonderheit, dass der Vertreter für die erstmalige Vertretung im Rahmen des Früherkennungsprogramms die Vorausset- zungen nach $\S 5$ Abs. 1 und Abs. 5b) bis e) und h) der Anlage 9.2 BMV-Ä zu erfüllen hat.

Für weitere Vertretungen müssen die Voraussetzungen zur Aufrechterhaltung und Weiterentwicklung der fachlichen Befähigung gemäß §24 Abs. 3 der Anlage 9.2 BMV-Ä erfüllt werden.

\section{Aufteilung vertragsärzt- licher Leistungen nach dem EBM}

Unter Beachtung der Ausführungen im ersten Teil dieses Beitrags zum Grundsatz der persönlichen Leistungserbringung, der zulässigen Beteiligung Dritter an der persönlichen Leistungserbringung sowie den zulässigen Orten der Leistungserbringung und den Erläuterungen in diesem zweiten Teil des Beitrags zu der zulässigen Leistungserbringung durch Vertreter, sind vertragsärztliche Leistungen durch den Vertragsarzt, die BAG oder das MVZ zulässigerweise erbring- sowie abrechenbar.

Die bisherigen Ausführungen bezogen sich jedoch - mit Ausnahme der Hinweise zur Delegation an nichtärztliches Personal auf vollständige vertragsärztliche Leistungen, die mit einer Gebührenordnungsposition (GOP) nach dem Einheitlichen Bewertungsmaßstab (EBM) abrechenbar sind, also die Erbringung einer Leistung durch einen Arzt an einem Standort.

In der radiologischen Praxis ist es aber üblich, dass einzelne vertragsärztliche radiologische Leistungen, die mit einer GOP abrechenbar sind, zwischen verschiedenen Ärzten, Standorten oder sogar Arztpraxen aufgeteilt werden.

Geht man der Einfachheit halber davon aus, dass sich eine radiologische Leistung in drei Teile teilen lässt, nämlich in

- rechtfertigende Indikation,

- technische Durchführung und

- Befundung,

ist es regelmäßig üblich, dass insbesondere die Befundung durch einen anderen Arzt zu einem anderen Zeitpunkt und an einem an- 
deren Standort, ggf. sogar in einer anderen Arztpraxis, als die anderen beiden Leistungsanteile erbracht wird.

Dies geschieht jedoch ohne Klärung der Frage, ob und in welchem Umfang dies in der vertragsärztlichen Versorgung zulässig ist; diese soll im Folgenden behandelt werden.

Die Besonderheiten der Teleradiologie, bei der ebenfalls eine Aufteilung der radiologischen Leistung zwischen verschiedenen Orten und Ärzten erfolgt, sollen dabei in diesem Beitrag unberücksichtigt bleiben.

\section{Leistungsaufteilung zwischen verschiedenen Ärzten}

Aus dem Grundsatz der persönlichen Leistungserbringung im Vertragsarztrecht folgt zunächst, dass eine vertragsärztliche radiologische Leistung nicht beliebig unter verschiedenen Ärzten aufgeteilt werden darf.

\section{Allgemeine Bestimmungen Ziffer 2.1 des} EBM sieht vor, dass eine GOP nur berechnungsfähig ist, wenn der Leistungsinhalt vollständig erbracht worden ist. I. Allgemeine Bestimmungen Ziffer 2.2 EBM regelt weiterhin, dass eine Gebührenordnungsposition nur berechnungsfähig ist, wenn der an der vertragsärztlichen Versorgung teilnehmende Arzt die für die Abrechnung relevanten Inhalte gemäß $\S \S 14 a, 15$ und 25 BMV-Ä persönlich erbringt.

Der Grundsatz der persönlichen Leistungserbringung wird in der BAG und im MVZ jedoch durchbrochen und modifiziert. Die Genehmigung der gemeinsamen Berufsausübung bzw. die Zulassung als MVZ bewirkt, dass die BAG bzw. das MVZ gegenüber der Kassenärztlichen Vereinigung wie ein Einzelarzt als einheitliche Rechtspersönlichkeit auftritt.

Zu der Abrechnung einer vertragsärztlichen Leistung, die durch mehrere Ärzte innerhalb einer Praxis, einer BAG oder eines MVZ erbracht worden ist, bestimmt I. Allgemeine Bestimmungen Ziffer 2.1 Satz 3 EBM Folgendes:

„Wirken an der Behandlung mehrere Ärzte zusammen, erfolgt die Berechnung durch denjenigen Vertragsarzt (Arztnummer), von dem die Vollständigkeit des Leistungsinhalts erreicht worden ist."

Der EBM geht also offenbar davon aus, dass die Aufteilung einer vertragsärztlichen Leistung, die mit einer GOP abrechenbar ist, zwischen verschiedenen Vertragsärzten zulässig ist. ${ }^{3}$

Die Zulässigkeit einer solchen Leistungsaufteilung wird auch von der Kassenärztlichen Bundesvereinigung bestätigt.

Der Kölner Kommentar zum EBM, der insoweit von Herrn Dr. Casser und damit vom Leiter des Dezernats Vergütung, Gebührenordnung und Morbiditätsorientierung bei der Kassenärztlichen Bundesvereinigung verfasst ist, geht ebenfalls von der Zulässigkeit der Aufteilung einer vertragsärztlichen Leistung zwischen verschiedenen Ärzten aus. ${ }^{4}$

Im Ergebnis wird es deshalb - obgleich dies nicht von sämtlichen Kassenärztlichen Vereinigungen ohne Weiteres anerkannt wird bspw. als zulässig anzusehen sein, wenn eine vertragsärztliche radiologische Leistung in der Weise aufgeteilt wird, dass rechtfertigende Indikation und technische Durchführung von Arzt A erbracht werden, die Befundung aber letztlich durch Arzt B erfolgt.

\section{Leistungsaufteilung zwischen verschiedenen Standorten}

Entsprechendes wird - obgleich auch dies nicht von sämtlichen Kassenärztlichen Vereinigungen ohne Weiteres anerkannt wird für die Aufteilung einer vertragsärztlichen radiologischen Leistung zwischen verschiedenen Standorten einer Praxis, einer BAG oder eines MVZ gelten.

Es wird bspw. als zulässig anzusehen sein, wenn eine vertragsärztliche radiologische Leistung in der Weise aufgeteilt wird, dass rechtfertigende Indikation und technische Durchführung an Standort A erbracht wer-

3 Dazu, weshalb dies nur eine vermeintlich eindeutige Regelung ist, soll sogleich im Zusammenhang mit der Frage nach der Zulässigkeit der Aufteilung einer vertragsärztlichen Leistung zwischen verschiedenen Arztpraxen eingegangen werden.

4 Vgl. Casser (Hrsg), Kölner Kommentar zum EBM, 2018, B1, I, Nr. 2.1. den, die Befundung jedoch an Standort B erfolgt.

Zwar enthält der EBM diesbezüglich keine ebenso eindeutige Regelung wie hinsichtlich der Leistungsaufteilung zwischen verschiedenen Ärzten, dabei ist jedoch zu berücksichtigen, dass den Formulierungen im EBM weiterhin die Vorstellung der Einzelpraxis mit nur einem Tätigkeitsort des Arztes zugrunde liegt und der Normgeber bei der Formulierung des EBM die Möglichkeiten der gemeinschaftlichen Berufsausübung an mehreren Standorten nicht berücksichtigt hat.

Die Kassenärztliche Bundesvereinigung bestätigt jedenfalls die Zulässigkeit der Aufteilung einer radiologischen Leistung zwischen verschiedenen Standorten und auch Casser geht im Kölner Kommentar zum EBM von der Zulässigkeit der Leistungsaufteilung zwischen verschiedenen Standorten aus. $^{5}$

Für diese Auffassung spricht weiterhin, dass die Allgemeinen Bestimmungen des EBM von der Möglichkeit einer arztpraxisübergreifenden Behandlung, also einer räumlich standortübergreifenden Leistungserbringung ausgehen. ${ }^{6}$

\section{Leistungsaufteilung zwischen verschiedenen Arztpraxen}

Aufgrund dieser Rede von einer „arztpraxisübergreifenden Behandlung“ und insbesondere vor dem Hintergrund der Regelungen zur sogenannten Leistungserbringergemeinschaft in $\S 15$ Abs. 3 BMV- ${ }^{7}$, könnte

5 Vgl. ebd.

6 Unter anderem ist dort von „arztpraxisübergreifender Behandlung“ und davon, dass „mehrere Arztpraxen“ an der Leistungserbringung mitgewirkt haben, die Rede.

7 §15 Abs. 3 S. 1 und 2 BMV-Ä lauten: „Vertragsärzte können sich bei gerätebezogenen Untersuchungsleistungen zur gemeinschaftlichen Leistungserbringung mit der Maßgabe zusammenschließen, dass die ärztlichen Untersuchungsleistungen nach fachlicher Weisung durch einen der beteiligten Ärzte persönlich in seiner Praxis oder in einer gemeinsamen Einrichtung durch einen gemeinschaftlich beschäftigten angestellten Arzt nach §32b Ärzte-ZV erbracht werden. Die Leistungen sind persönliche Leistungen des jeweils anweisenden Arztes, der an der Leistungsgemeinschaft beteiligt ist." 
man davon ausgehen, dass auch die Aufteilung radiologischer Leistungen zwischen verschiedenen Arztpraxen als zulässig anzusehen ist, zumal Casser im Kölner Kommentar zum EBM ebenfalls von der Zulässigkeit einer derartigen Leistungsaufteilung auszugehen scheint. ${ }^{8}$

Eine Aufteilung vertragsärztlicher Leistungen zwischen verschiedenen Arztpraxen wird von einigen Kassenärztlichen Vereinigungen zudem durchaus als zulässig akzeptiert. Eine als zulässig anerkannte arztpraxisübergreifende Leistungsaufteilung kommt beispielsweise in der Form vor, dass ein Orthopäde, der über die erforderliche Fachkunde im Strahlenschutz verfügt, zunächst die rechtfertigende Indikation selbst stellt, die technische Durchführung einer Röntgenuntersuchung von dem benachbarten Radiologen vornehmen lässt, die gefertigten Aufnahmen hingegen selbst befundet.

Eine derartige Aufteilung vertragsärztlicher Leistungen bedeutet allerdings eine ganz erhebliche Durchbrechung des Grundsatzes der persönlichen Leistungserbringung.

Die Rechtsberatertagung bei der Kassenärztlichen Bundesvereinigung ist wohl deshalb im Juni des vergangenen Jahres zu der Rechtsauffassung gelangt, dass die Allgemeinen Bestimmungen des EBM, soweit sie von der Zulässigkeit einer arztpraxisübergreifenden Leistungsaufteilung ausgehen, nur Abrechnungsregelungen sind, aus denen nicht hergeleitet werden kann, dass eine derartige Leistungsaufteilung generell zulässig ist. Vielmehr müsse die Zulässigkeit einer arztpraxisübergreifenden Leistungsaufteilung in der jeweiligen GOP geregelt sein. ${ }^{9}$

Von der Zulässigkeit der arztpraxisübergreifenden Aufteilung einer vertragsärztlichen radiologischen Leistung ist deshalb keinesfalls ohne Weiteres auszugehen. Ohne vorherige Abstimmung mit der zuständigen Kassenärztlichen Vereinigung und der rechtssicheren vertraglichen Vereinbarung einer derartigen Kooperation sollte eine arztpraxisübergreifende Leistungsauftei-

\footnotetext{
8 Vgl. Casser (Hrsg), ebd.

$9 \quad$ Eine GOP mit einer entsprechenden Regelung ist im EBM in der aktuellen Fassung hingegen nicht enthalten.
}

lung nicht praktiziert werden, um sich nicht dem Vorwurf einer Falschabrechnung ausgesetzt zu sehen.

\section{Kennzeichnung und Abrechnung der aufgeteilten Leistungen}

Eine GOP ist nach dem EBM nur berechnungsfähig, wenn der Leistungsinhalt vollständig erbracht worden ist, vgl. I. Allgemeine Bestimmungen Ziffer 2.1 EBM.

Die Vollständigkeit der Leistungserbringung ist gegeben, wenn die erforderlichen Leistungsinhalte erbracht worden und die erbrachten Leistungen dokumentiert sind. Die in der Überschrift zu einer GOP aufgeführten Leistungsinhalte sind immer Bestandteil der obligaten Leistungsinhalte.

GOPen, deren Leistungsinhalt nicht vollständig erbracht wurde, können nicht berechnet werden. Eine GOP ist nur berechnungsfähig, wenn der an der vertragsärztlichen Versorgung teilnehmende Arzt die für die Abrechnung relevanten Inhalte gemäß $\S \S 14 a, 15$ und 25 BMV-Ä persönlich erbringt, vgl. I. Allgemeine Bestimmungen Ziffer 2.2 EBM.

Bei der Aufteilung einer vertragsärztlichen Leistung zwischen verschiedenen Ärzten, Standorten und, soweit zulässig, Arztpraxen gilt, dass die Leistungen mit der LANR des Arztes zu kennzeichnen sind, von dem die Vollständigkeit des Leistungsinhalts erreicht worden ist und dass die (Neben-)Betriebsstättennummer des Standorts anzugeben ist, in dem die Vollständigkeit des Leistungsinhalts erreicht worden ist, vgl. I. Allgemeine Bestimmungen Ziffer 2.1 EBM sowie die Abrechnungsrichtlinien der Kassenärztlichen Vereinigungen.

Aus diesen Abrechnungsvorschriften ergeben sich jedoch Folgeprobleme, die bei der Aufteilung vertragsärztlicher Leistungen zu beachten sind und ggf. zu einer Begrenzung des Umfangs der Leistungsaufteilung führen.

Insbesondere, wenn bei der Aufteilung vertragsärztlicher radiologischer Leistungen einzelne Ärzte überwiegend nicht mehr mit der Befundung oder im Gegenteil überwiegend mit der Befundung betraut sind oder an einzelnen Standorten überwiegend befundet oder im Gegenteil kaum noch befundet wird, können die Folgenden Probleme auftreten:

Diejenigen Ärzte, die kaum noch vertragsärztliche radiologische Leistungen befunden, erfüllen den ihnen übertragenen Versorgungsauftrag rechnerisch nicht mehr, weil die Leistungen, an deren Erbringung sie mitwirken, nicht mit ihrer LANR zu kennzeichnen sind. Nach § 19 Abs. 4 Ärzte-ZV in der seit dem 11.05.2019 geltenden Fassung kann dies in letzter Konsequenz dazu führen, dass die Kassenärztliche Vereinigung die Zulassung in dem Umfang, in dem der Versorgungsauftrag nicht erfüllt wird, zu entziehen hat.

Hingegen werden die Zeitprofile derjenigen Ärzte, die überwiegend die Befundung der vertragsärztlichen radiologischen Leistungen übernehmen, in einer Plausibilitätsprüfung in erheblichem Maße auffällig sein, weil sämtliche Leistungen, an deren Erbringung sie durch die Befundung beteiligt sind, mit ihrer LANR zu kennzeichnen sind. Ob und in welchem Umfang eine Plausibilisierung der deshalb zeitauffälligen Abrechnungen im Rahmen der Plausibilitätsprüfung möglich ist, hängt dann letztlich von der Verwaltungspraxis der zuständigen Kassenärztlichen Vereinigung ab.

Bei der Aufteilung einer vertragsärztlichen Leistung zwischen verschiedenen Standorten sind zudem $\S 17$ Abs. 1 a S. 3 und 4 BMV-Ä zu beachten. Nach deren Vorschriften muss die Tätigkeit am Vertragsarztsitz alle Tätigkeiten außerhalb des Vertragsarztsitzes zeitlich insgesamt überwiegen (Überwiegenheitsgebot), wobei bei MVZ nicht auf den einzelnen im MVZ tätigen Arzt, sondern auf das MVZ insgesamt abzustellen ist. Erfolgt die Befundung vertragsärztlicher radiologischer Leistungen nun überwiegend nicht am Vertragsarztsitz, ist auch mit einer grundsätzlich zulässigen Leistungsaufteilung schnell ein Verstoß gegen das Überwiegenheitsgebot des BMV-Ä verbunden. 


\section{Fazit}

Wird ein Vertragsarzt oder ein angestellter Arzt, der an der vertragsärztlichen Versorgung teilnimmt, von einem anderen an der vertragsärztlichen Versorgung teilnehmenden Arzt derselben Praxis, derselben BAG oder desselben MVZ vertreten, ist stets abzugrenzen, ob es sich bei dieser internen Vertretung um „echte“ Vertretung im Sinne der Ärzte-ZV oder um sogenannte „unechte“ Vertretung handelt. Diese Abgrenzung ist dafür maßgeblich, ob die Leistung als Vertreterleistung abzurechnen ist, mit wessen LANR sie zu kennzeichnen ist und über welche Abrechnungsgenehmigungen der Vertretende verfügen muss. Die Abgrenzung zwischen „echter“ und „unechter“ Vertretung bereitet jedoch immer wieder Schwierigkeiten und hängt nach der insoweit uneinheitlichen Verwaltungspraxis der Kassenärztlichen Vereinigungen oftmals von Detailfragen ab.
Die Aufteilung vertragsärztlicher Leistungen zwischen verschiedenen Ärzten und verschiedenen Standorten einer Praxis, einer BAG oder eines MVZ dürfte grundsätzlich zulässig sein. Sicherheitshalber sollte vor einer entsprechenden Aufteilung vertragsärztlicher Leistungen zwischen verschiedenen Standorten und Ärzten das Einvernehmen mit der zuständigen Kassenärztlichen Vereinigung erzielt werden.

Die Aufteilung einer vertragsärztlichen Leistung zwischen verschiedenen Arztpraxen sollte nur in enger Abstimmung mit der zuständigen Kassenärztlichen Vereinigung und auf Grundlage klarer vertraglicher Bestimmungen erfolgen, um sich nicht dem Vorwurf der Falschabrechnung ausgesetzt zu sehen.

In jedem Fall der Aufteilung vertragsärztlicher Leistungen zwischen verschiedenen Ärzten, Standorten oder, soweit zulässig,
Arztpraxen sind die Grenzen zu beachten, die sich aus dem Erfordernis der Erfüllung des Versorgungsauftrags, aus der Verwaltungspraxis der zuständigen Kassenärztlichen Vereinigung im Hinblick auf die Möglichkeiten der Plausibilisierung auffälliger Zeitprofile in der Plausibilitätsprüfung und aus dem Überwiegenheitsgebot des BMVÄ ergeben.

Prof. Dr. Peter Wigge

Rechtsanwalt

Fachanwalt für Medizinrecht

Jonas Kaufhold

Rechtsanwalt

Rechtsanwälte Wigge

Scharnhorststraße 40

48151 Münster

Telefon: (0251) $53595-0$

Telefax: (0251) 53595 - 99

E-Mail: kanzlei@ra-wigge.de www.ra-wigge.de 